\title{
Quantum decoherence effects in neutrino oscillations at DUNE
}

\author{
G. Balieiro Gomes $\odot,{ }^{1,2, *}$ D. V. Forero, ${ }^{1,3, \dagger}$ M. M. Guzzo, ${ }^{1, \$}$ P. C. de Holanda, ${ }^{1, \S}$ and R. L. N. Oliveira ${ }^{1,4, \|}$ \\ ${ }^{1}$ Instituto de Física Gleb Wataghin Universidade Estadual de Campinas-UNICAMP, \\ Rua Sérgio Buarque de Holanda, 777 13083-970 Campinas, São Paulo, Brazil \\ ${ }^{2}$ Instituto Federal de Educação, Ciência e Tecnologia de São Paulo, 13565-905 São Carlos, SP, Brazil \\ ${ }^{3}$ Universidad de Medellín, Carrera $87 N^{\circ} 30$ - 65 Medellín, Colombia \\ ${ }^{4}$ Universidade Federal do ABC-UFABC, Santo André, 09210-580 São Paulo, Brazil
}

(Received 29 April 2019; published 17 September 2019)

\begin{abstract}
In this work, we analyze quantum decoherence in neutrino oscillations considering the open quantum system framework and oscillations through matter for three-neutrino families. Taking the Deep Underground Neutrino Experiment as a case study, we performed sensitivity analyses for two neutrino flux configurations, finding sensitivity limits for the decoherence parameters. We also offer a physical interpretation for a new peak which arises at the $\nu_{e}$ appearance probability with decoherence. The sensitivity limits found for the decoherence parameters are $\Gamma_{21} \leq 1.2 \times 10^{-23} \mathrm{GeV}$ and $\Gamma_{32} \leq$ $7.7 \times 10^{-25} \mathrm{GeV}$ at $90 \%$ C.L.
\end{abstract}

DOI: $10.1103 /$ PhysRevD.100.055023

\section{INTRODUCTION}

Even though the standard three-neutrino oscillation paradigm is well established and several oscillation parameters have been already measured with certain precision [1], the quest for establishing the violation of the charge parity $(C P)$ symmetry in the leptonic sector, the octant preference or the maximality of the atmospheric mixing angle, and the neutrino mass ordering is still ongoing. In order to fulfill such goals and also to reach a greater precision in the measurement of all the neutrino oscillation parameters, future experiments such as the Deep Underground Neutrino Experiment (DUNE) [2-6] are being developed. DUNE is a long-baseline neutrino experiment where the neutrinos produced at Fermilab are detected at the Sanford Underground Research Laboratory, therefore after traveling $\sim 1300 \mathrm{~km}$. DUNE is designed to study the $\nu_{\mu}$ and $\nu_{e}$ (and also $\bar{\nu}_{\mu}$ and $\bar{\nu}_{e}$ ) oscillations through Earth's crust matter, and it is expected to provide a measurement of the neutrino mass hierarchy. DUNE is also sensitive to the Dirac phase present in the lepton mixing matrix, which parameterizes the possibility that neutrinos violate the $C P$ symmetry.

\footnotetext{
*balieiro@ifi.unicamp.br

†vanegas@udem.edu.co *guzzo@ifi.unicamp.br

holanda@ifi.unicamp.br

"robertol@ifi.unicamp.br
}

Published by the American Physical Society under the terms of the Creative Commons Attribution 4.0 International license. Further distribution of this work must maintain attribution to the author(s) and the published article's title, journal citation, and DOI. Funded by SCOAP ${ }^{3}$.
In order to perform these major discoveries and the precise measurement of the atmospheric mixing angle, DUNE will have to reach a novel control of systematics and very large statistics. Such features can be used not only to achieve the main goals for the standard oscillation program, but, more importantly, can also be useful to probe new physics effects, such as decoherence.

There are several works [7-17] showing how decoherence can emerge in models considering interactions between a neutrino subsystem and an environment in the open quantum system [18] framework, and some of these works present analyses of possible constraints for the decoherence parameters $[9,15,17]$. Nevertheless, there are other experiments which could be considered and might be suitable to make a full three-neutrino family analysis. As will be shown later on, the decoherence effect arises in the oscillation probabilities through damping terms depending on the baseline, suggesting that a long-baseline experiment such as DUNE is an excellent candidate to bound all the decoherence parameters for three-neutrino families. Although it is speculated that the quantum decoherence effect could be generated by quantum gravity [19], in this work we will use a phenomenological approach. We do not use any microscopical model which describes the source of such effects, and, therefore, such a hypothesis or other possible origins of decoherence will not be discussed. It is also important to point out that in this work we will study only the decoherence effects which arise in the framework of open quantum systems; we will not address decoherence effects from wave packet separation (see, for example, Refs. [20,21]), which are already present within usual quantum mechanics. 
This work is organized in the following way. We review how one can study neutrino oscillations considering a coupling with the environment in the quantum open system framework in Sec. II, presenting also the form of the oscillation probabilities with decoherence in three families. In Sec. III, we offer a physical interpretation of a new peak that arises in the oscillation probabilities with decoherence. In Sec. IV, we perform sensitivity analyses and present the sensitivity regions found for the decoherence parameters. Since the optimized flux configuration at DUNE already covers a broad range of neutrino energies, DUNE is sensitive to the decoherence parameters. We also consider a high-energy flux configuration to reach the high-energy peak induced by decoherence in the appearance channel, which is the "smoking gun" for decoherence, providing increased sensitivity to the decoherence parameter $\Gamma_{32}$.

\section{FORMALISM}

When the coupling between the neutrino subsystem and the environment is considered, the time evolution of the subsystem density operator $\rho$ is given by the Lindblad master equation [11]:

$$
\frac{d}{d t} \rho(t)=L \rho=-i[H, \rho]+\frac{1}{2} \sum_{k=1}^{N^{2}-1}\left(\left[V_{k}, \rho V_{k}^{\dagger}\right]+\left[V_{k} \rho, V_{k}^{\dagger}\right]\right),
$$

where $H$ is the subsystem's Hamiltonian, $V_{k}$ are the operators responsible for the interactions between the subsystem and the environment, and $N$ is the dimension of the Hilbert space of the subsystem. The sum that runs from $k=1$ to $\left(N^{2}-1\right)$ denotes an expansion on $S U(N)$ generators, with the element $k=0$ decoupled from the evolution due to constraints on the operators $V_{k}$ which are briefly mentioned below. The non-Hamiltonian term can be written as

$$
D[\rho(t)]=\frac{1}{2} \sum_{k=1}^{N^{2}-1}\left(\left[V_{k}, \rho V_{k}^{\dagger}\right]+\left[V_{k} \rho, V_{k}^{\dagger}\right]\right),
$$

which will be referred from now on as the dissipator.

The matrix $D$ is subjected to constraints to assure that the operator $\rho(t)$ has all the properties of a density operator and that its physical interpretation is correct. In particular, it can be shown that the operator $V$ must be Hermitian $\left(V_{k}=V_{k}^{\dagger}\right)$ [7], to ensure that the system's entropy increases in time.

In the case of three active neutrinos, one can expand the elements on the Lindblad equation in Eq. (1) using the $S U(3)$ generators, the Gell-Mann matrices $\lambda_{i}$, as a basis:

$$
H=H_{i} \lambda_{i} ; \quad \rho=\rho_{j} \lambda_{j},
$$

where the sum over repeated indices is implied, and Eq. (1) can be rewritten as

$$
\frac{d}{d t} \rho_{k}(t) \lambda_{k}=f^{i j k} H_{i} \rho_{j}(t) \lambda_{k}+D_{k l} \rho_{l} \lambda_{k}
$$

where the $f^{i j k}$ are structure constants completely antisymmetric in the indices $i, j$, and $k$.

We assume $D_{k l}$ as a symmetric matrix and with $D_{k 0}=$ $D_{0 l}=0$ in order to have probability conservation. We will also impose that $\left[H, V_{k}\right]=0$, which implies energy conservation in the neutrino subsystem. Other conditions for $D$ will come from the imposition that it satisfies the criteria for complete positivity, which must be obeyed by a density operator and, hence, also by the dissipator. For threeneutrino families, these criteria are described in Ref. [7] and references therein. Although the derivation of these conditions can be found in these references, we found it worthwhile to present them again in Appendix A for the specific case analyzed here, namely, with energy conservation in the neutrino sector.

Under such constraints, the dissipative matrix $D_{k l}$ assumes the following form:

$$
D_{k l}=-\operatorname{diag}\left\{\Gamma_{21}, \Gamma_{21}, 0, \Gamma_{31}, \Gamma_{31}, \Gamma_{32}, \Gamma_{32}, 0\right\} .
$$

The decoherence parameters are not independent from each other and are related by the following equations [13]:

$$
\begin{gathered}
\Gamma_{21}=2 a_{3}^{2} \geq 0 ; \\
\Gamma_{31}=\frac{1}{2}\left(a_{3}+a_{8}\right)^{2} \geq 0 ; \\
\Gamma_{32}=\frac{1}{2}\left(a_{3}-a_{8}\right)^{2} \geq 0,
\end{gathered}
$$

where the $a_{i}$ are the coefficients of the expansion of the $V_{k}$ operators in terms of the $S U(3)$ matrix basis:

$$
V_{k}=a_{n}^{k} \lambda_{n}
$$

Since we have that a density matrix must be positive semidefinite, which means that if $\lambda_{i}$ are its eigenvalues, then $\lambda_{i} \geq 0 \forall i$ [22], it is clear that the dissipator in Eq. (4) with the conditions in Eqs. (5)-(7) satisfies the needed criteria in order to preserve its physical meaning. In Appendix B, we discuss the validity of the dissipator in Eq. (4) and the positivity conditions when one considers decoherence in a vacuum or in constant density matter, highlighting the differences between our approach and the one used in Ref. [16]. In the following sections, we consider $\Gamma_{21}$ and $\Gamma_{32}$ as the independent parameters and $\Gamma_{31}$ given by Eqs. (5)-(7).

Considering the DUNE baseline, matter effects have to be taken into account. The complete Hamiltonian in the flavor basis is then given by 


$$
H=\left[U\left(\begin{array}{ccc}
0 & 0 & 0 \\
0 & \frac{\Delta m_{21}^{2}}{2 E} & 0 \\
0 & 0 & \frac{\Delta m_{31}^{2}}{2 E}
\end{array}\right) U^{\dagger}+\left(\begin{array}{ccc}
\hat{A} & 0 & 0 \\
0 & 0 & 0 \\
0 & 0 & 0
\end{array}\right)\right]
$$

where the $\Delta m_{i j}^{2} \equiv m_{i}^{2}-m_{j}^{2}$ are the squared mass differences between the mass eigenstates, $E$ is the neutrino energy, $\hat{A}=$ $\sqrt{2} G_{F} n_{e}$ is the matter potential, where $G_{F}$ is the Fermi coupling constant and $n_{e}$ is the electron number density, and $U$ is the mixing matrix for three-neutrino families, which is given by

$$
U=\left(\begin{array}{ccc}
c_{12} c_{13} & s_{12} c_{13} & s_{13} e^{-i \delta_{C P}} \\
-s_{12} c_{23}-c_{12} s_{23} s_{13} e^{i \delta_{C P}} & c_{12} c_{23}-s_{12} s_{23} s_{13} e^{i \delta_{C P}} & s_{23} c_{13} \\
s_{12} s_{23}-c_{12} c_{23} s_{13} e^{i \delta_{C P}} & -c_{12} s_{23}-s_{12} c_{23} s_{13} e^{i \delta_{C P}} & c_{23} c_{13}
\end{array}\right),
$$

and where $c_{i j}$ and $s_{i j}$ denote $\cos \left(\theta_{i j}\right)$ and $\sin \left(\theta_{i j}\right)$, respectively.

Equation (3) will be solved in the effective mass eigenstate basis; hence, we must find the diagonal form of the Hamiltonian:

$$
H=\frac{1}{2 E}\left(\begin{array}{ccc}
0 & 0 & 0 \\
0 & \tilde{\Delta}_{21} & 0 \\
0 & 0 & \tilde{\Delta}_{31}
\end{array}\right)
$$

where $\tilde{\Delta}_{i j}$ are the effective squared mass differences of neutrinos in matter.

Solving the Lindblad equation, with the dissipator defined in Eq. (4), one finds

$$
\rho_{\tilde{m}}(x)=\left(\begin{array}{ccc}
\rho_{11}(0) & \rho_{12}(0) e^{-\left(\Gamma_{21}+i \tilde{\Delta}_{21}\right)^{*} x} & \rho_{13}(0) e^{-\left(\Gamma_{31}+i \tilde{\Delta}_{31}\right)^{*} x} \\
\rho_{21}(0) e^{-\left(\Gamma_{21}+i \tilde{\Delta}_{21}\right) x} & \rho_{22}(0) & \rho_{23}(0) e^{-\left(\Gamma_{32}+i \tilde{\Delta}_{32}\right)^{*} x} \\
\rho_{31}(0) e^{-\left(\Gamma_{31}+i \tilde{\Delta}_{31}\right) x} & \rho_{32}(0) e^{-\left(\Gamma_{32}+i \tilde{\Delta}_{32}\right) x} & \rho_{33}(0)
\end{array}\right)
$$

where $\rho_{i j}(0)$ are the elements of the density matrix for the initial state.

The oscillation probabilities for each channel can be calculated from

$$
P_{\nu_{\alpha} \nu_{\beta}}=\operatorname{Tr}\left[\rho_{\alpha}(0) \rho_{\beta}(x)\right]
$$

Using Eqs. (12) and (13), and after some algebraic manipulation, one obtains

$$
\begin{aligned}
P_{\nu_{\alpha} \nu_{\beta}}= & \delta_{\alpha \beta}-2 \sum_{j>k} \operatorname{Re}\left(\tilde{U}_{\beta j} \tilde{U}_{\alpha j}^{*} \tilde{U}_{\alpha k} \tilde{U}_{\beta k}^{*}\right) \\
& +2 \sum_{j>k} \operatorname{Re}\left(\tilde{U}_{\beta j} \tilde{U}_{\alpha j}^{*} \tilde{U}_{\alpha k} \tilde{U}_{\beta k}^{*}\right) e^{-\Gamma_{j k} x} \cos \left(\frac{\tilde{\Delta}_{j k}}{2 E} x\right) \\
& +2 \sum_{j>k} \operatorname{Im}\left(\tilde{U}_{\beta j} \tilde{U}_{\alpha j}^{*} \tilde{U}_{\alpha k} \tilde{U}_{\beta k}^{*}\right) e^{-\Gamma_{j k} x} \sin \left(\frac{\tilde{\Delta}_{j k}}{2 E} x\right),
\end{aligned}
$$

where $\tilde{U}$ is the unitary mixing matrix which diagonalizes the Hamiltonian in the presence of matter effects. To obtain the corresponding probability for antineutrinos, one must repeat the procedure above, changing $\hat{A} \rightarrow-\hat{A}$ in Eq. (9) and $\delta_{C P} \rightarrow-\delta_{C P}$ in Eq. (10). It is also important to point out that we assumed the decoherence parameters $\Gamma_{j k}$ as being equal for both neutrinos and antineutrinos.

In the following sections, we present results from the implementation of Eq. (14) in a modified version of the GLoBES $[23,24]$ probability engine, which was also double checked by solving numerically the Lindblad equation in Eq. (3).

\section{EFFECTS OF DECOHERENCE ON THE OSCILLATION PROBABILITIES}

We consider the four oscillation channels, appearance and disappearance for both neutrino and antineutrino modes, for benchmark values of the decoherence parameters $\Gamma_{21}, \Gamma_{31}$, and $\Gamma_{32}$. For the probability studies, only the DUNE baseline $(L=1300 \mathrm{~km})$ and its energy range (which extends from hundreds of $\mathrm{MeV}$ to tens of $\mathrm{GeV}$ ) are needed. The values of the standard oscillation parameters used along this work are given in Table I.

As we can see in Fig. 1, the decoherence parameters affect the four oscillation channels, and for the values of the decoherence parameters considered we can see a few different effects on the oscillation probabilities. In Figs. 1(c) 
TABLE I. Values for the standard oscillation parameters from Refs. [1,25,26].

\begin{tabular}{lc}
\hline \hline $\sin ^{2} \theta_{12}$ & 0.321 \\
$\sin ^{2}\left(2 \theta_{13}\right)$ & 0.0841 \\
$\sin ^{2}\left(2 \theta_{23}\right)$ & 0.99 \\
$\delta_{C P}$ & $-\pi / 2$ \\
$\Delta m_{21}^{2}$ & $7.56 \times 10^{-5} \mathrm{eV}^{2}$ \\
$\Delta m_{31}^{2}$ & $2.55 \times 10^{-3} \mathrm{eV}^{2}$ \\
\hline \hline
\end{tabular}

and 1(d), there is a small decrease in the overall oscillation amplitude (more accentuated for the $\bar{\nu}_{\mu}$ disappearance probability). In Fig. 1(d), we can also see a decrease in the $\bar{\nu}_{\mu}$ for $E \gtrsim 10 \mathrm{GeV}$. However, the most striking difference with respect to the standard oscillation is the new peak at $\sim 10 \mathrm{GeV}$ for the $\nu_{e}$ appearance probability in the presence of decoherence, which would provide a clear signature of new physics. In the next section, we will discuss this feature in more detail. Although the peak by itself is not a novelty, and it was somehow studied in previous works (see, for instance, $[13,14])$, here we provide a detailed physical interpretation,

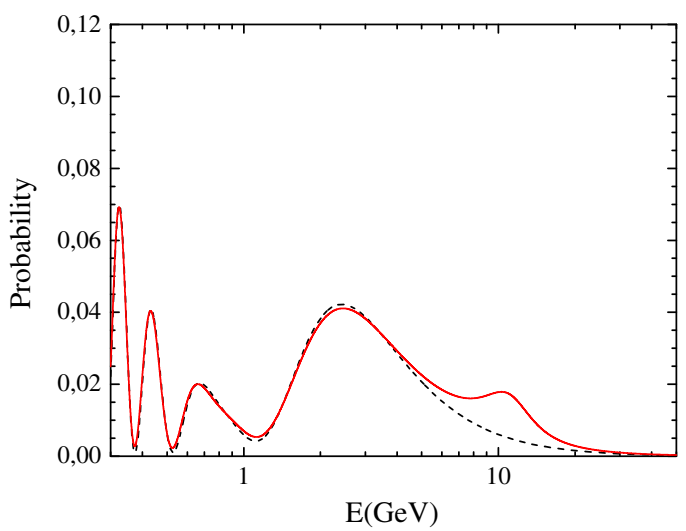

(a) $\nu_{e}$ appearance

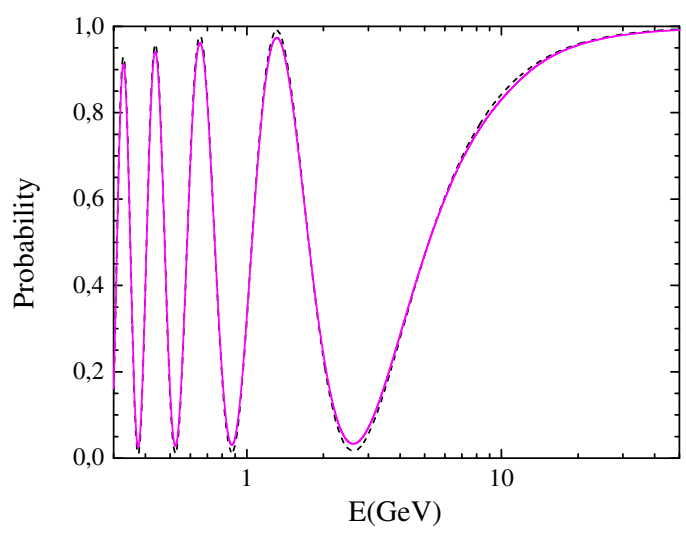

(c) $\nu_{\mu}$ disappearance and, more importantly, we suggest how this unique feature of decoherence can be probed at DUNE.

\section{A. A new peak at the $\nu_{e}$ appearance probability: Physical interpretation}

A peak at $\sim 10 \mathrm{GeV}$ is present in the $\nu_{e}$ appearance probability in the presence of decoherence. In order to obtain a physical insight of this new feature, let us begin by analyzing the behavior of the eigenvalues of the Hamiltonian $(\lambda)$ in Eq. (9), which can be seen in the upper panel in Fig. 2.

As we can see in Fig. 2, there is a level crossing between the eigenvalues referred as 2 and 3 at $E \sim 10 \mathrm{GeV}$, which indicates a resonance at that energy for the parameters considered. By numerically diagonalizing the Hamiltonian, it is possible to calculate the oscillation parameters in matter $\tilde{U}_{\alpha j}$ and $\tilde{\Delta}_{j k}$. In particular, at the resonance energy, and for the DUNE baseline, we obtain

$$
\tilde{U}_{e 1} \simeq 0, \quad \frac{\tilde{\Delta}_{32}}{2 E} x \ll 1,
$$

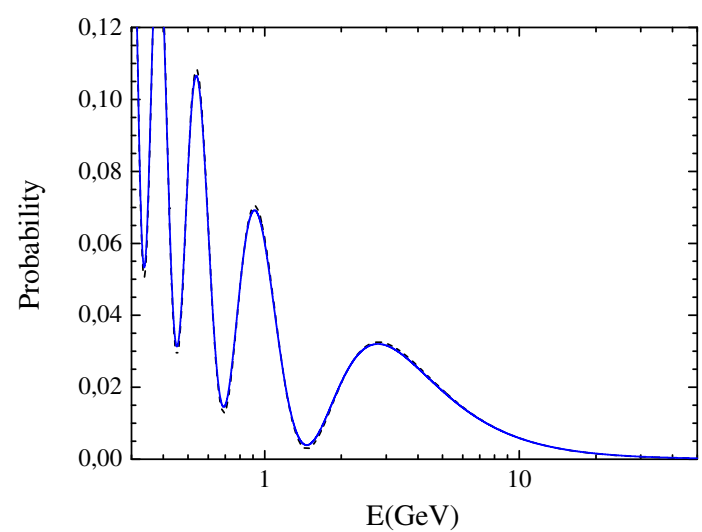

(b) $\bar{\nu}_{e}$ appearance

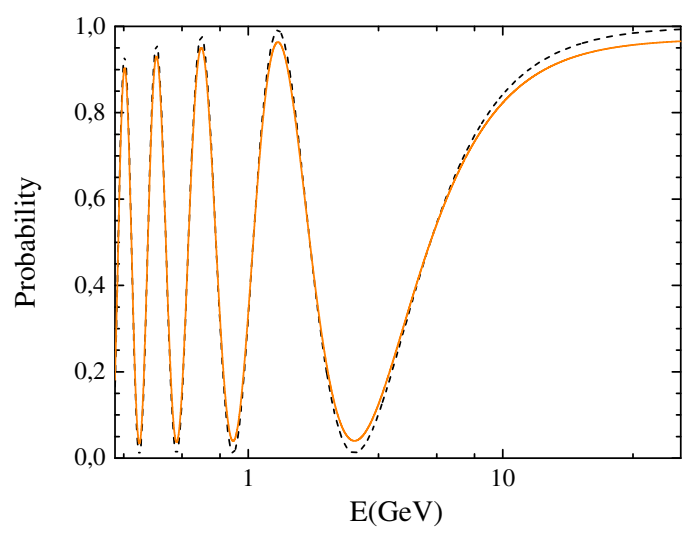

(d) $\bar{\nu}_{\mu}$ disappearance

FIG. 1. Oscillation probabilities using $\Gamma_{21}=5.1 \times 10^{-25} \mathrm{GeV}$ and $\Gamma_{32}=8.9 \times 10^{-24} \mathrm{GeV}$ (solid line) compared with the standard case without decoherence (dashed line). The values of the oscillation parameters were set according to Table I, and $\Gamma_{31}$ was calculated according to Eqs. (5)-(7). 


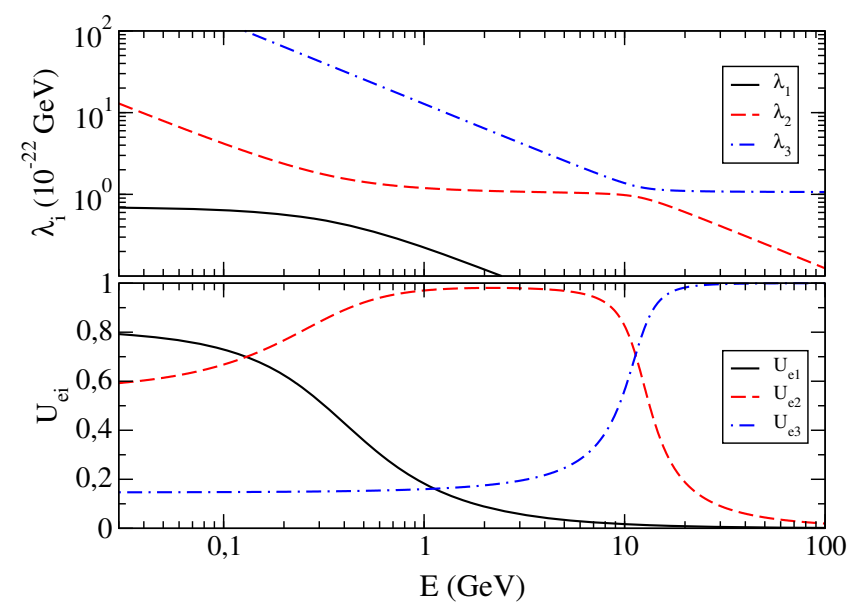

FIG. 2. Eigenvalues $\left(\lambda_{i}, i=1,2,3\right)$ of the Hamiltonian in Eq. (9). We can see an indication of a resonance region at about $E \sim 10 \mathrm{GeV}$.

where the first approximation can be extracted directly from the lower panel in Fig. 2 while the second one is obtained by replacing $x \sim 1300 \mathrm{~km} \sim 6.5 \times 10^{21} \mathrm{GeV}^{-1}$ and the values calculated in upper panel in Fig. 2 for the $\lambda_{i}$ 's. From the oscillation probabilities with decoherence in Eq. (14), the $\Gamma_{j k}$ parameters appear in the form of $e^{-\Gamma_{j k} x}$ damping factors for the terms:

$$
\begin{aligned}
I^{j k}(\alpha \beta) \equiv & 2 \operatorname{Re}\left(\tilde{U}_{\beta j} \tilde{U}_{\alpha j}^{*} \tilde{U}_{\alpha k} \tilde{U}_{\beta k}^{*}\right) \cos \left(\frac{\tilde{\Delta}_{j k}}{2 E} x\right) \\
& +2 \operatorname{Im}\left(\tilde{U}_{\beta j} \tilde{U}_{\alpha j}^{*} \tilde{U}_{\alpha k} \tilde{U}_{\beta k}^{*}\right) \sin \left(\frac{\tilde{\Delta}_{j k}}{2 E} x\right) .
\end{aligned}
$$

Since $I^{j k}(\alpha \beta)$ is the term of the probability where we have the dependence on the oscillation phase through $\cos \left(\frac{\tilde{\Delta}_{j k}}{2 E} x\right)$ and $\sin \left(\frac{\tilde{\Delta}_{j k}}{2 E} x\right)$, which are responsible for the quantum interference in the oscillation probabilities, we will refer to it as the interference factor. In addition, there are terms not affected by the decoherence parameters:

$$
C^{j k}(\alpha \beta) \equiv-2 \operatorname{Re}\left(\tilde{U}_{\beta j} \tilde{U}_{\alpha j}^{*} \tilde{U}_{\alpha k} \tilde{U}_{\beta k}^{*}\right),
$$

and the flavor conversion probability in Eq. (14) can be written as

$$
P_{\nu_{\alpha} \nu_{\beta}}=\delta_{\alpha \beta}+\sum_{j>k}\left[C^{j k}(\alpha \beta)+I^{j k}(\alpha \beta) e^{-\Gamma_{j k} x}\right]
$$

In the case of the $\nu_{e}$-appearance probability at DUNE, the only contribution for both $I$ and $C$ at the resonance comes from the case $j=3$ and $k=2$ :

$C^{32}(\mu e)=-2 \operatorname{Re}\left(\tilde{U}_{e 3} \tilde{U}_{\mu 3}^{*} \tilde{U}_{\mu 2} \tilde{U}_{e 2}^{*}\right) \simeq 2\left|\tilde{U}_{e 3}\right|^{2}\left|\tilde{U}_{\mu 3}\right|^{2}$,

$I^{32}(\mu e) \simeq-2\left|\tilde{U}_{e 3}\right|^{2}\left|\tilde{U}_{\mu 3}\right|^{2} \simeq-C^{32}(\mu e)$,
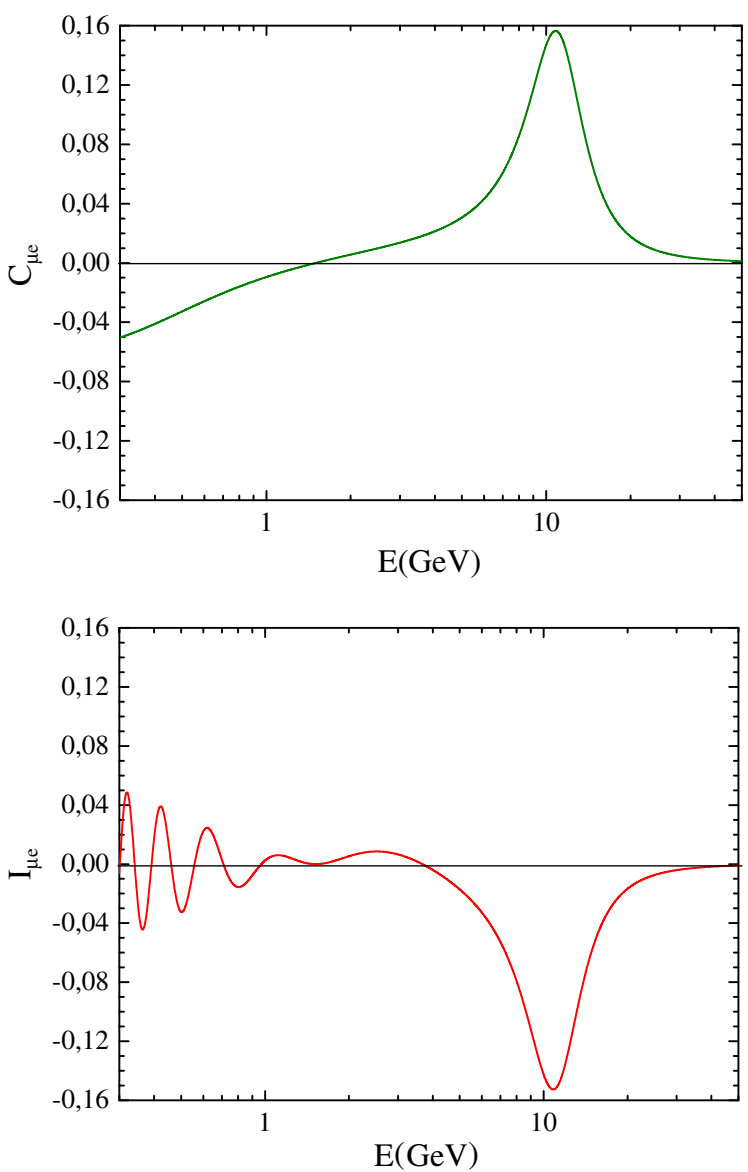

FIG. 3. Behavior of $C^{32}(\mu e)$ and the interference factor $I^{32}(\mu e)$ given by Eqs. (17) and (16), respectively.

where the relations in Eq. (15) and the unitarity of the mixing matrix were used.

The form of the interference term, which is subject to the damping factor $e^{-\Gamma_{32} x}$, and as given in Eq. (19), is shown in Fig. 3. We can see that it works as an interference factor to the oscillation probabilities; in particular, we see that around $E=10.8 \mathrm{GeV}$, which is exactly the energy of the resonance, we have a strong destructive interference. For the standard oscillation probabilities (without decoherence), such destructive interference would be exactly canceled at the resonance $E \sim 10 \mathrm{GeV}$. In fact, considering a constant matter density of $2.96 \mathrm{~g} / \mathrm{cm}^{3}$ [27,28], numerically the maximum of $C^{32}(\mu e)$ (upper panel) is equal to the minimum of $I^{32}(\mu e)$ (lower panel), and both coincide at $E=10.82 \mathrm{GeV}$. However, when we have oscillations with nonzero decoherence, the term $e^{-\Gamma_{32} x}$ works as a damping to this interference factor, therefore eliminating the destructive interference at $E \sim 10 \mathrm{GeV}$. The elimination of such destructive interference enhances the $\nu_{e}$ appearance probability, since now the destructive interference cannot completely be canceled the resonance, therefore creating the peak shown in Fig. 1(a). Since such a peak constitutes a very significant effect in the oscillation 
probabilities in the presence of decoherence, being able to reconstruct it will provide a compelling test of decoherence. If DUNE is compatible with standard oscillations, severe bounds to the decoherence parameters can be obtained as long as the experiment measures a significant number of events around $E \sim 10 \mathrm{GeV}$.

Rewriting Eqs. (18) and (19) in terms of the mixing angles, we would have

$$
P_{\mu e}\left(E=E_{\mathrm{res}}\right) \approx \frac{1}{2} \sin ^{2} \theta_{23} \sin ^{2}\left(2 \tilde{\theta}_{13}\right)\left(1-e^{-\Gamma_{32} x}\right),
$$

and then we expect that the effects from $\Gamma_{32}$ on the bump would anticorrelate with $\theta_{23}$. Changing $\Delta m_{32}^{2}$ would displace the resonance energy, and then the effect would be stronger for lower values of $\Delta m_{32}^{2}$, which would locate the bump in probability at lower energies. However, in the sensitivity analysis, we marginalized over the atmospheric parameters within their current experimental range, taking care of the correlations between the relevant parameters.

\section{RESULTS}

In this section, we are going to show sensitivity analyses considering neutrino oscillations with decoherence in matter given by Eq. (14). We first show how each oscillation channel is sensible to decoherence by calculating the event rates, and then we establish DUNE sensitivities to the decoherence parameters. For the sensitivity analysis, we have considered two neutrino flux configurations, as will be detailed in the following sections, to exploit the main features of the decoherence effects discussed previously.

In the following studies, we assume the DUNE configuration as defined in the conceptual design report (CDR) document in Ref. [3], and, in particular, we made use of the GLoBES files from Ref. [29]. Basically, it is assumed DUNE will be running for $3.5 \mathrm{yr}$ in each mode (neutrino and antineutrino), a fiducial mass of the far detector (liquid argon) of $40 \mathrm{kt}$, and the default flux beam power of 1.07 MW. The channels considered in each analysis are defined for each case. The systematical errors, energy resolution, and efficiencies are fixed to the values in the CDR studies.

\section{A. Relative events with the DUNE default flux configuration}

For a particular input of the decoherence parameters, the total number of events and the energy event spectra are calculated for each oscillation channel. We define the relative event rates as $\delta R_{\text {rel }}$ :

$$
\delta R_{\text {rel }}=\frac{R\left(\Gamma_{i j} \neq 0\right)-R\left(\Gamma_{21}=\Gamma_{31}=\Gamma_{32}=0\right)}{R\left(\Gamma_{21}=\Gamma_{31}=\Gamma_{32}=0\right)},
$$

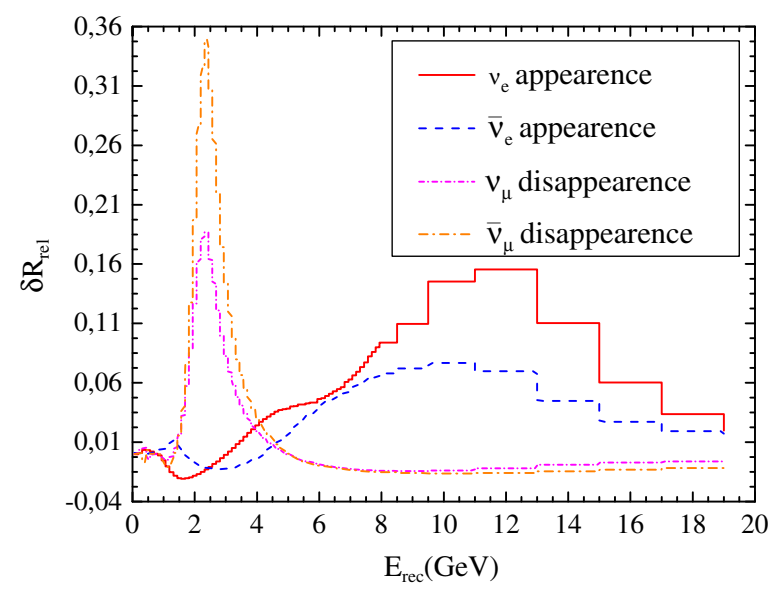

FIG. 4. Relative event rates defined in Eq. (20) setting $\Gamma_{21}=5.1 \times 10^{-25} \mathrm{GeV}$, and $\Gamma_{32}=8.9 \times 10^{-24} \mathrm{GeV}$, for each oscillation channel in the presence of decoherence.

where $\Gamma_{i j} \neq 0$ (i.e., $\Gamma_{21} \neq 0, \Gamma_{31} \neq 0$, and $\Gamma_{32} \neq 0$ ) are chosen in order to satisfy Eqs. (5)-(7) and $R$ correspond to the event rates.

Figure 4 shows the relative deviation of the number of $\nu_{e}$, $\bar{\nu}_{e}, \nu_{\mu}$, and $\bar{\nu}_{\mu}$ events with respect to the standard oscillation case without decoherence. From the $\nu_{e}$ and $\bar{\nu}_{e}$ events, one can see a low relative deviation $(<3 \%)$ at the DUNE flux (default) maximum $(\sim 2.5 \mathrm{GeV})$. The peak at $E \gtrsim 10 \mathrm{GeV}$ in the $\nu_{e}\left(\bar{\nu}_{e}\right)$ events is also relatively low, being about $\sim 16 \%$ ( $\sim 8 \%)$, but this is expected, because, with the default flux, events at the high energy end of the spectrum are much smaller than in the DUNE energy peak. In the case of $\nu_{\mu}$ and $\bar{\nu}_{\mu}$ events in Fig. 4, we can notice that, at slightly lower energies from the DUNE flux (default) maximum, a relative deviation of the order of $\sim 19 \%$ is obtained in the case of $\nu_{\mu}$ and $\sim 35 \%$ for $\bar{\nu}_{\mu}$ events.

It appears to be that, with the default flux configuration, DUNE is sensitive to decoherence, and this sensitivity is obtained from the four oscillation channels. However, due to the large number of muon neutrino (and antineutrino) events (see Table II) and the relative deviation in Fig. 4, the main sensitivity comes from $\nu_{\mu}$ and $\bar{\nu}_{\mu}$ events and some reduced sensitivity from $\bar{\nu}_{e}$ events. To fully exploit the high-energy relative deviations that appear in the $\nu_{e}$ and $\bar{\nu}_{e}$ events in Fig. 4, a high-energy flux for DUNE will be considered in the sensitivity analysis of Sec. IV C, and, as will be shown, this will substantially improve the sensitivity for testing decoherence.

TABLE II. Total number of events (signal plus background) for each oscillation channel.

\begin{tabular}{lccc}
\hline \hline$\nu_{e}$-app & $\bar{\nu}_{e}$-app & $\nu_{\mu}$-disapp & $\bar{\nu}_{\mu}$-disapp \\
\hline 1777.69 & 406.025 & 8206.77 & 4124.51 \\
\hline \hline
\end{tabular}




\section{B. DUNE sensitivity to the decoherence parameters with the default flux configuration}

In this section, we present a sensitivity analysis considering the default flux configuration from Ref. [29]. From the previous sections, we could see that with the default flux DUNE has a good sensitivity to the decoherence parameters $\Gamma_{21}$ and $\Gamma_{32}$ in a parameter range which is not yet constrained by other experiments. Later, we present a second analysis considering a higher-energy flux, which will bring a better sensitivity to $\Gamma_{32}$, since it is the parameter which generates the new peak at $\sim 10 \mathrm{GeV}$ for the $\nu_{e}$ appearance probability.

For the analysis presented in this section, we have assumed the DUNE energy-event spectrum is given by the standard oscillation "data" without decoherence, from the values in Table I, and the decoherence hypothesis is tested. The usual $\chi^{2}$ analysis has been performed, marginalizing over the standard oscillation parameters (except for the solar parameters that are kept fixed) adding penalties to the $\chi^{2}$ function with the following standard deviations: $\sigma\left(\sin ^{2}\left(2 \theta_{13}\right)\right)=0.0033, \sigma\left(\sin ^{2}\left(2 \theta_{23}\right)\right) / \sin ^{2}\left(2 \theta_{23}\right)=3 \%$, and $\sigma\left(\Delta m_{31}^{2}\right) / \Delta m_{31}^{2}=3 \%$. The $\delta$ parameter has been also marginalized over. Because $\Gamma_{21}, \Gamma_{31}$, and $\Gamma_{32}$ are not all independent, to perform the $\chi^{2}$ analysis, we assumed two of the three decoherence parameters as independent and defined the other one as a dependent parameter, according to Eqs. (5)-(7), making then confidence level curves shown in Fig. 5.

To obtain the sensitivity regions on each individual parameter, we marginalize over one of the two decoherence parameters. In the upper (lower) panel in Fig. 6, the $\Gamma_{32}$ $\left(\Gamma_{21}\right) \Delta \chi^{2}$ profile is shown. From the profiles, we calculated the sensitivity regions compiled in Table III.

It is worthwhile to compare the achievable bounds by DUNE, obtained in this work, with current bounds coming from other experiments. In Table IV, we present the main

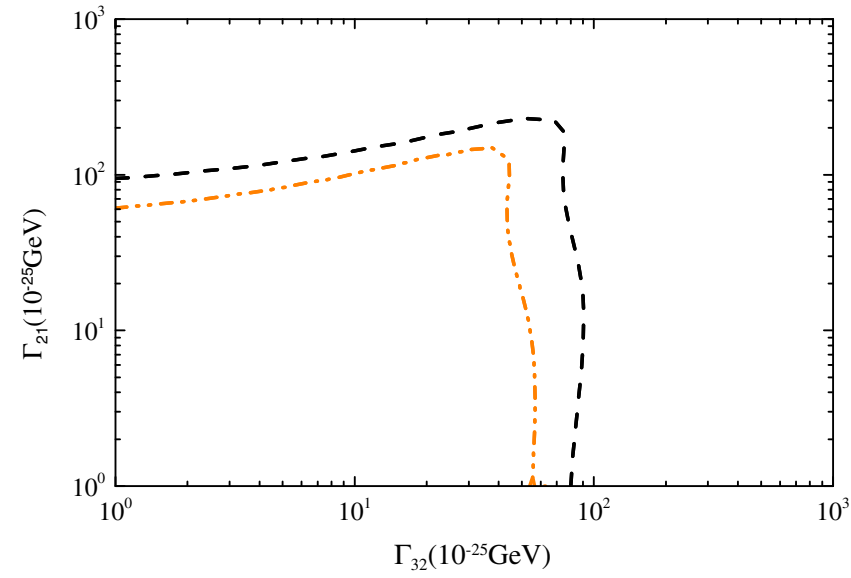

FIG. 5. Curves at $90 \%$ C.L. (dashed-dotted line) and $3 \sigma$ C.L. (dashed line) for 2 DOF for the two decoherence parameters $\Gamma_{21}$ and $\Gamma_{32}$, considering the default flux given by Ref. [29].

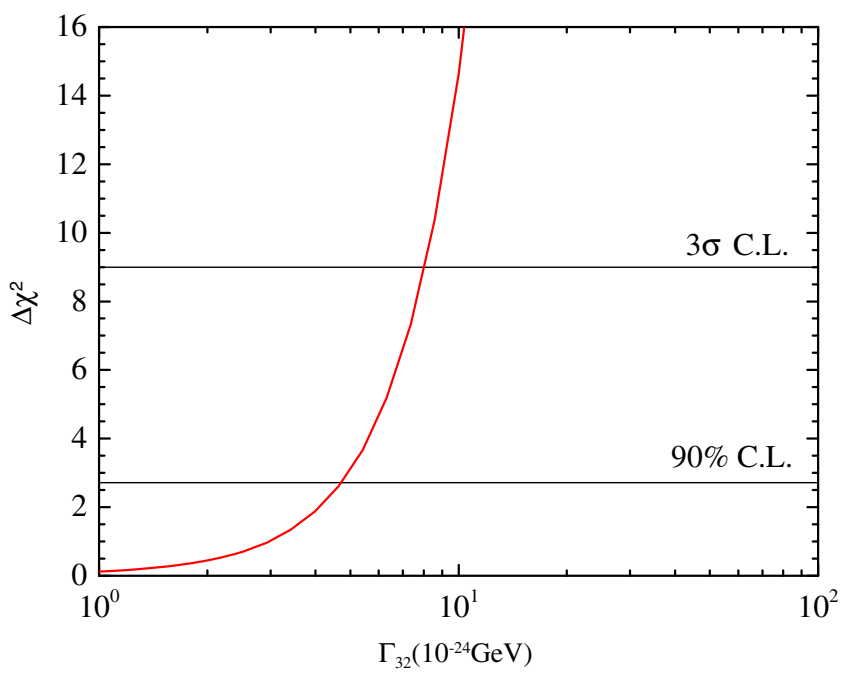

(a) $\Delta \chi^{2}$ versus $\Gamma_{32}$, minimizing over $\Gamma_{21}$

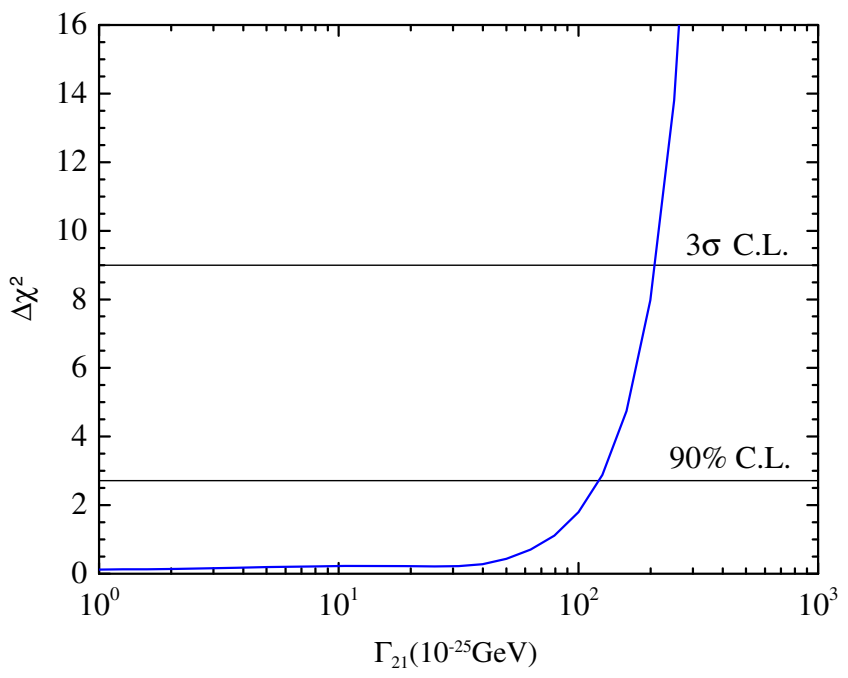

(b) $\Delta \chi^{2}$ versus $\Gamma_{21}$, minimizing over $\Gamma_{32}$

FIG. 6. Sensitivity limits for the default flux in Ref. [29] for the $\Gamma_{32}\left(\Gamma_{21}\right)$ upper (lower) panel. The horizontal lines define $90 \%$ C.L. and $3 \sigma$ C.L.

bounds coming from a recent analysis of data from IceCube [17], MINOS [15], and KamLAND [9]. Although these bounds are not directly comparable due to different assumptions and marginalizations, it is possible to state that DUNE has the potential to provide a more stringent limit to $\Gamma_{21}$. However, for $\Gamma_{32}$ and $\Gamma_{31}$ the limits obtainable

TABLE III. Sensitivity regions for the decoherence parameters from the $\chi^{2}$ analysis considering the default flux configuration [29], as shown in Figs. 6(a) and 6(b) for 1 DOF.

\begin{tabular}{lcc}
\hline \hline Parameter & $90 \%$ C.L. & $3 \sigma$ C.L. \\
\hline$\Gamma_{21} \leq$ & $1.2 \times 10^{-23} \mathrm{GeV}$ & $2.1 \times 10^{-23} \mathrm{GeV}$ \\
$\Gamma_{32} \leq$ & $4.7 \times 10^{-24} \mathrm{GeV}$ & $8.0 \times 10^{-24} \mathrm{GeV}$ \\
\hline \hline
\end{tabular}


TABLE IV. Existing limits at 95\% C.L. coming from different analyses and data.

\begin{tabular}{lcc}
\hline \hline Experiments & Assumptions on $\Gamma$ 's & $\begin{array}{c}\text { Previous bounds } \\
\left(\mathrm{GeV}^{-1}\right)\end{array}$ \\
\hline MINOS [15] & 2-fam, $\Gamma_{32}$ or $\Gamma_{31}$ & $1.1 \times 10^{-22}$ \\
KamLAND [9] & 2 -fam, $\Gamma_{21}$ & $6.8 \times 10^{-22}$ \\
IceCube [17] & $\Gamma_{31}=\Gamma_{32}\left(\Gamma_{21}=0\right)$ & $4.0 \times 10^{-24}$ \\
IceCube [17] & $\Gamma_{31}=\Gamma_{21}\left(\Gamma_{32}=0\right)$ & $1.3 \times 10^{-24}$ \\
\hline \hline
\end{tabular}

by DUNE would be competitive with existing ones coming from an analysis of IceCube data [17].

In the following section, we discuss how a high-energy neutrino flux, different from the one given by Ref. [29], can considerably improve the sensitivity to $\Gamma_{32}$, providing a more suitable configuration to test decoherence at DUNE.

\section{Sensitivity analysis for $\Gamma_{32}$ with a high-energy flux configuration}

From the discussion at the event level in Sec. IVA, it is clear that, in order to be sensitive to the peak around $10 \mathrm{GeV}$ in the $\nu_{e}$ appearance channel at DUNE, it is necessary to consider a different flux configuration. Having reached this conclusion, we decided to perform a second sensitivity analysis, but this time considering the highenergy (HE) neutrino flux proposed in Ref. [30].

For the sensitivity analysis using the HE flux, we excluded the beam contamination from $\nu_{e}$ and $\bar{\nu}_{e}$, since we do not have access to this information. Then, we repeated the same procedure of the previous sections, first presenting in Fig. 7 the relative deviation of the number of

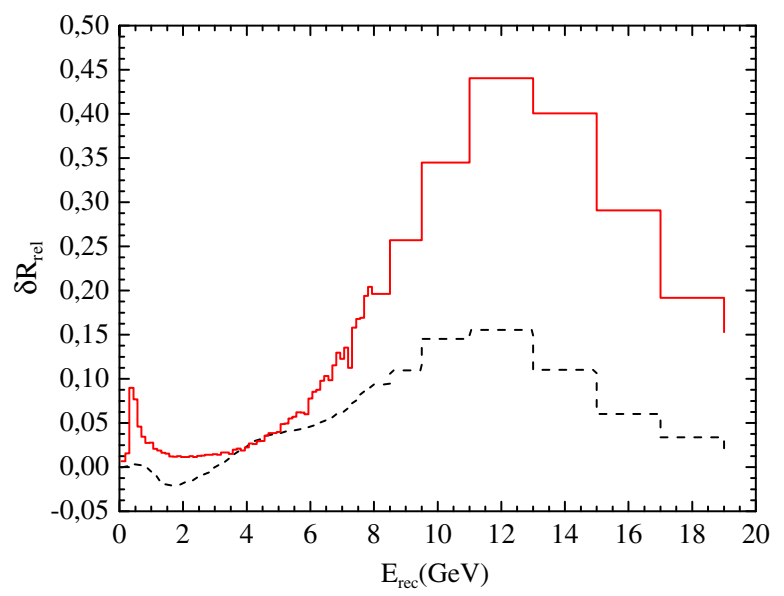

FIG. 7. Relative deviations with respect to the $\nu_{e}$ appearance events without decoherence $\left(\Gamma_{32}=0\right)$ for the HE flux from Ref. [30] (red line). For the event rates with decoherence, we considered $\Gamma_{21}=5.1 \times 10^{-25} \mathrm{GeV}, \quad \Gamma_{31}=3.0 \times 10^{-25} \mathrm{GeV}$, and $\Gamma_{32}=1.6 \times 10^{-24} \mathrm{GeV}$. We also present the relative deviations for the $\nu_{e}$ appearance events considering the default flux [29] for comparison (black dashed line).

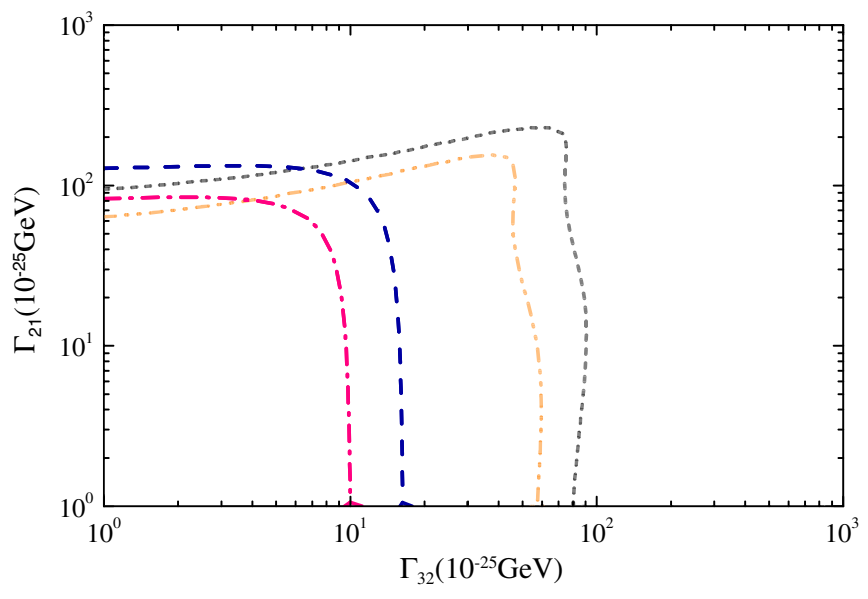

FIG. 8. Confidence level curves in the $\Gamma_{32}-\Gamma_{21}$ plane at $90 \%$ C.L. (dark dashed-dotted line) and $3 \sigma$ C.L. (dark dashed line), considering the HE flux. The confidence level curves for the default flux (light colors) are also shown for comparison (see Fig. 5).

$\nu_{e}$ events with respect to the standard oscillation case without decoherence and, finally, the sensitivity results. As already expected, with the HE flux configuration the peak in the $\nu_{e}$ rises to $\sim 45 \%$, which suggests that this channel with such a flux configuration can bring increased sensitivity to the $\Gamma_{32}$ parameter. We showed in Sec. III that it is the $\Gamma_{32}$ parameter that mostly generates this new peak. Following the same procedure of the previous section, we performed another sensitivity analysis, and the results are given in Figs. 8 and 9.

From Fig. 9, we obtained the sensitivity regions compiled in Table V, where we present only the limits for $\Gamma_{32}$, since the analysis presented in Sec. IV B already brings the

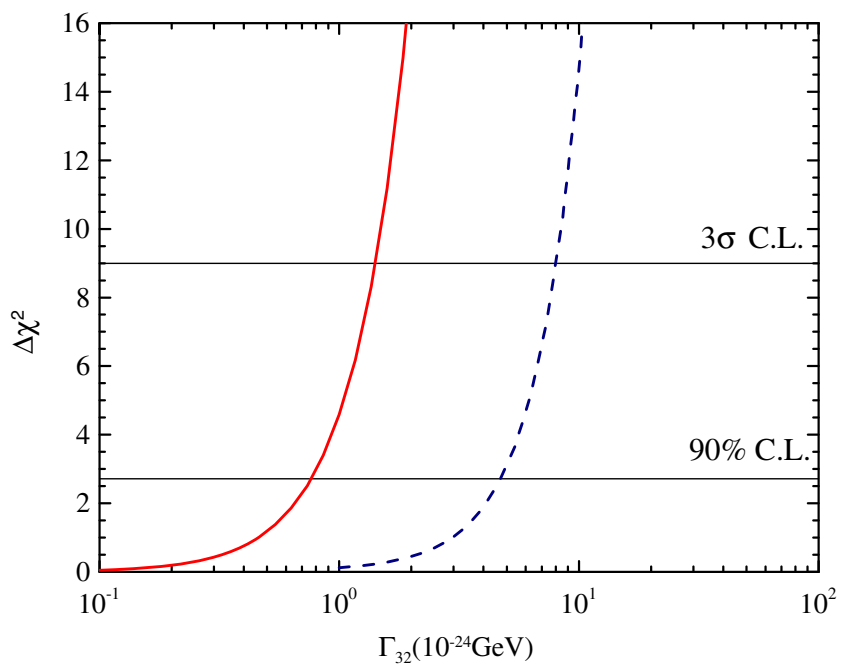

FIG. 9. $\quad \Gamma_{32} \chi^{2}$ profile after minimizing over $\Gamma_{21}$ for the results with the default flux (dashed line) from Ref. [29] and the ones with HE flux (full line) from Ref. [30]. The horizontal lines define $90 \%$ C.L. and $3 \sigma$ C.L. 
TABLE V. Sensitivities to the decoherence parameters from the $\chi^{2}$ analysis considering the HE flux configuration from Ref. [30] shown in Fig. 9 for 1 DOF.

\begin{tabular}{lcc}
\hline \hline Parameter & $90 \%$ C.L. & $3 \sigma$ C.L. \\
\hline$\Gamma_{32} \leq$ & $7.7 \times 10^{-25} \mathrm{GeV}$ & $1.4 \times 10^{-24} \mathrm{GeV}$ \\
\hline \hline
\end{tabular}

best sensitivity to $\Gamma_{21}$. The sensitivity to $\Gamma_{32}$ given in Table V is enhanced respect to the one found in Sec. IV B, since the HE flux from Ref. [30] is much more suitable to pin down the new peak at $E \sim 10.8 \mathrm{GeV}$ in the $\nu_{e}$ appearance probability than the default flux [29]. DUNE has the potential to put a stringent limit to the decoherence parameters, which means that the peak would become much less noticeable in a $\nu_{e}$ appearance probability plot as long as the DUNE measured rates are more "compatible" with standard oscillations.

\section{CONCLUSION}

In this work, we obtained sensitivity regions for the decoherence parameters that affect neutrino oscillations in three families considering two possible flux configurations for DUNE.

In Sec. III, we showed how the new peak at the $\nu_{e}$ appearance probability can be seen as an elimination of a destructive interference, generating then an increase in the transition to $\nu_{e}$. In Sec. IV, we showed how the decoherence parameters can be better analyzed by considering different oscillation channels and also different flux configurations, the default flux from Ref. [29] and the HE flux from Ref. [30].

In Sec. IV B, we presented the results for the sensitivity analysis using the flux configuration from Ref. [29]. In $90 \%$ C.L., the sensitivity limits for the parameters given in Table III are $\Gamma_{21} \leq 1.2 \times 10^{-23} \mathrm{GeV}$ and $\Gamma_{32} \leq$ $4.7 \times 10^{-24} \mathrm{GeV}$, and for $3 \sigma$ C.L. the limits are $\Gamma_{21} \leq$ $2.1 \times 10^{-23} \mathrm{GeV}$ and $\Gamma_{32} \leq 8.0 \times 10^{-24} \mathrm{GeV}$.

As we can see, the limits on $\Gamma_{21}$ are potentially more stringent at DUNE, when compared with the KamLAND experiment, by 2 orders of magnitude. On the other hand, DUNE in its default configuration has a reduced sensitivity for $\bar{\nu}_{e}$, suggesting that the limit for $\Gamma_{21}$ comes in most part from the $\nu_{e}$ channel. Therefore, one might think that $\Gamma_{21}$ for $\nu_{e}$ and $\bar{\nu}_{e}$ has some chance to be different. This is the exact scenario for a $C P T$-like violation such as was proposed in Ref. [31], and a new investigation regarding such an issue will be presented somewhere else.

Finally, in Sec. IV C, we showed how, by changing to a HE flux configuration, DUNE can significantly improve the sensitivity to the $\Gamma_{32}$ parameter, potentially pinning down the peak which is the most compelling feature of decoherence at DUNE. The sensitivity regions for such an analysis (presented in Table V) are $\Gamma_{32} \leq 7.7 \times 10^{-25} \mathrm{GeV}$ at $90 \%$ C.L. and $\Gamma_{32} \leq 1.4 \times 10^{-24} \mathrm{GeV}$ at $3 \sigma$ C.L.

\section{ACKNOWLEDGMENTS}

The authors thank FAPESP, CAPES, and CNPq for several financial supports. M. M. G. and P. C. H. are grateful for the support of FAPESP funding Grant No. 2014/ 19164-6 and CNPq research fellowships No. 304001/20171 and No. 310952/2018-2, respectively. D. V. F. is thankful for the support of the São Paulo Research Foundation (FAPESP) funding Grants No. 2014/19164-6, No. 2017/ 01749-6, and No. 2018/19365-2.

\section{APPENDIX A: CONDITIONS ON $D_{\mu \nu}$}

The Lindblad equation that describes the open system's dynamics is given by

$$
\frac{d \rho(t)}{d t}=-i[H, \rho(t)]+\frac{1}{2} \sum_{j=1}^{8}\left(\left[V_{j}, \rho(t) V_{j}^{\dagger}\right]+\left[V_{j} \rho(t), V_{j}^{\dagger}\right]\right),
$$

where $V_{j}$ are $3 \times 3$ matrices that carry out the new dynamics. Expanding in Gell-Mann matrices in the mass eigenbasis, where $H$ is diagonal, we have

$$
H=h_{3} \lambda_{3}+h_{8} \lambda_{8} ; \quad V_{j}=\sum_{i=1}^{8} v_{j i} \lambda_{i} ; \quad \rho=\sum_{i=1}^{8} \rho_{i} \lambda_{i},
$$

where, since $V_{j}$ is Hermitian, all coefficients are real. The energy conservation condition $\left[V_{j}, H\right]=0$ leads to

$$
\begin{aligned}
{\left[V_{j}, H\right] } & =\sum_{i}\left(h_{3} v_{j i}\left[\lambda_{i}, \lambda_{3}\right]+h_{8} v_{j i}\left[\lambda_{i}, \lambda_{8}\right]\right) \\
& =2 i \sum_{i, k} v_{j i}\left(h_{3} f_{i 3 k}+h_{8} f_{i 8 k}\right) \lambda_{k}=0 .
\end{aligned}
$$

The only way to accomplish this with no dependence on $h_{3,8}$ is that all $v_{j i}$ vanishes except for $v_{j 3}$ and $v_{j 8}$. Then

$$
V_{j}=v_{j 3} \lambda_{3}+v_{j 8} \lambda_{8}
$$

By replacing in the Lindblad equation, we obtain 


$$
\begin{aligned}
\frac{d \rho_{k}(t)}{d t} \lambda_{k}= & 2 h_{m} \rho_{n} f_{m n k} \lambda_{k}+\frac{1}{2} \sum_{j=1}^{8} \sum_{k=1}^{8} \sum_{l=3,8} \sum_{m=3,8} \rho_{k} v_{j l} v_{j m}^{*}\left(\left[\lambda_{l}, \lambda_{k} \lambda_{m}^{\dagger}\right]+\left[\lambda_{l} \lambda_{k}, \lambda_{m}^{\dagger}\right]\right) \\
= & 2 h_{m} \rho_{n} f_{m n k} \lambda_{k}+\frac{1}{2}\left(\sum_{j}\left|v_{j 3}\right|^{2}\right) \sum_{k} \rho_{k}\left(\left[\lambda_{3}, \lambda_{k} \lambda_{3}^{\dagger}\right]+\left[\lambda_{3} \lambda_{k}, \lambda_{3}^{\dagger}\right]\right)+\frac{1}{2}\left(\sum_{j}\left|v_{j 8}\right|^{2}\right) \sum_{k} \rho_{k}\left(\left[\lambda_{8}, \lambda_{k} \lambda_{8}^{\dagger}\right]+\left[\lambda_{8} \lambda_{k}, \lambda_{8}^{\dagger}\right]\right) \\
& +\frac{1}{2}\left(\sum_{j} v_{j 3} v_{j 8}^{*}\right) \sum_{k} \rho_{k}\left(\left[\lambda_{3}, \lambda_{k} \lambda_{8}^{\dagger}\right]+\left[\lambda_{3} \lambda_{k}, \lambda_{8}^{\dagger}\right]\right)+\frac{1}{2}\left(\sum_{j} v_{j 3}^{*} v_{j 8}\right) \sum_{k} \rho_{k}\left(\left[\lambda_{8}, \lambda_{k} \lambda_{3}^{\dagger}\right]+\left[\lambda_{8} \lambda_{k}, \lambda_{3}^{\dagger}\right]\right) .
\end{aligned}
$$

Performing the last sum by direct inspection, we get

$$
\begin{aligned}
I_{1}^{k} & =\left[\lambda_{3}, \lambda_{k} \lambda_{3}\right]+\left[\lambda_{3} \lambda_{k}, \lambda_{3}\right] \\
& =\lambda_{3}\left[\lambda_{k}, \lambda_{3}\right]+\left[\lambda_{3}, \lambda_{k}\right] \lambda_{3} \\
& =2 i\left(\lambda_{3} \sum_{l} f_{k 3 l} \lambda_{l}+\sum_{l} f_{3 k l} \lambda_{l} \lambda_{3}\right) \\
& =2 i \sum_{l} f_{3 k l}\left[\lambda_{l}, \lambda_{3}\right]=-4 \sum_{l m} f_{3 k l} f_{l 3 m} \lambda_{m} \\
& =-4 \lambda_{k} \sum_{l}\left(f_{3 k l}\right)^{2} \\
\sum_{k} \rho_{k} I_{1}^{k} & =-(4,4,0,1,1,1,1,0) \cdot\left(\rho_{k} \lambda_{k}\right),
\end{aligned}
$$

and with a similar procedure

$$
\begin{aligned}
I_{2}^{k} & =\left[\lambda_{8}, \lambda_{k} \lambda_{8}\right]+\left[\lambda_{8} \lambda_{k}, \lambda_{8}\right]=-4 \lambda_{k} \sum_{l}\left(f_{8 k l}\right)^{2}, \\
\sum_{k} \rho_{k} I_{2}^{k} & =-3(0,0,0,1,1,1,1,0) \cdot\left(\rho_{k} \lambda_{k}\right) .
\end{aligned}
$$

The last two lines can be simplified, since $v_{i j}$ are real numbers. In this case, the last two lines can be summed up:

$$
\begin{aligned}
I_{3}^{k} & =\left[\lambda_{3}, \lambda_{k} \lambda_{8}\right]+\left[\lambda_{3} \lambda_{k}, \lambda_{8}\right]+(3 \leftrightarrow 8) \\
& =-8 \lambda_{k} \sum_{l} f_{8 k l} f_{3 k l}, \\
\sum_{k} \rho_{k} I_{3}^{k} & =-2 \sqrt{3}(0,0,0,1,1,-1,-1,0) .\left(\lambda_{k} \rho_{k}\right) .
\end{aligned}
$$

Defining the eight-dimensional vectors $\vec{a}_{k}$ formed by the components $v_{j k}$ in the following way:

$$
\left(\vec{a}_{3}\right)_{j} \equiv v_{j 3}, \quad\left(\vec{a}_{8}\right)_{j} \equiv \sqrt{3} v_{j 8}
$$

we have

$\sum_{j}\left|v_{j 3}\right|^{2}=a_{3}^{2} ; \quad \sum_{j}\left|v_{j 8}\right|^{2}=\frac{a_{8}^{2}}{3} ; \quad \sum_{j}\left(v_{j 3} v_{j 8}^{*}\right)=\frac{1}{\sqrt{3}}\left(\vec{a}_{3}^{\dagger} \cdot \vec{a}_{8}\right)$,

and we can finally write $\frac{d \rho(t)}{d t}=-i[H, \rho(t)]+\frac{1}{2} \sum_{k} \rho_{k}\left(a_{3}^{2} I_{1}^{k}+a_{8}^{2} \frac{I_{2}^{k}}{3}+2 \vec{a}_{3}^{\dagger} \cdot \vec{a}_{8} \frac{I_{3}^{k}}{2 \sqrt{3}}\right)$

and

$$
\begin{aligned}
D_{m n}= & -\frac{1}{2} \operatorname{diag}\left(4 a_{3}^{2}, 4 a_{3}^{2}, 0,\left(\vec{a}_{3}+\vec{a}_{8}\right)^{2},\left(\vec{a}_{3}+\vec{a}_{8}\right)^{2},\right. \\
& \left.\left(\vec{a}_{3}-\vec{a}_{8}\right)^{2},\left(\vec{a}_{3}-\vec{a}_{8}\right)^{2}, 0\right)
\end{aligned}
$$

or

$$
D_{m n}=-\operatorname{diag}\left(\Gamma_{21}, \Gamma_{21}, 0, \Gamma_{31}, \Gamma_{31}, \Gamma_{32}, \Gamma_{32}, 0\right),
$$

where

$\Gamma_{21} \equiv 2 a_{3}^{2} ; \quad \Gamma_{31} \equiv \frac{1}{2}\left(\vec{a}_{3}+\vec{a}_{8}\right)^{2} ; \quad \Gamma_{32} \equiv \frac{1}{2}\left(\vec{a}_{3}-\vec{a}_{8}\right)^{2}$.

For simplicity, we treated $\vec{a}_{3}$ and $\vec{a}_{8}$ as collinear, so they were treated as scalars. So the conclusion is that the matrix energy conservation in the neutrino sector requires a diagonal format for $D_{m n}$, with a specific relation between its terms.

\section{APPENDIX B: DECOHERENCE IN MATTER AND POSITIVITY}

The authors of Ref. [16] claim that decoherence cannot be defined in the effective mass basis and that (apart from very specific cases) the forms of the dissipative matrices in a vacuum and in matter cannot be the same. In this Appendix, we comment that, under certain conditions, decoherence can be defined as arising from the same matrices in both contexts, such that it preserves a physical interpretation where the decoherence effect acts only on the quantum interference terms, such as was discussed in this paper and also in Refs. [8,9].

The dissipator in Eq. (4) is obtained when it is imposed that

$$
\left[H_{S}, V_{k}\right]=0
$$

where $H_{S}$ is the Hamiltonian of the subsystem. 
Since the subsystem is different when one considers neutrinos in a vacuum or in matter, if $V_{k}$ has the same form in both bases, then Eq. (B1) is not satisfied at the same time in such cases. This is exactly what happens in Refs. $[10,16]$. As is shown in Ref. [8], this implies that the decoherence effect and the so-called relaxation effect cannot be fully separated. In fact, as argued in Refs. [8,9], the work in Ref. [10] finds constraints for the relaxation effect or for decoherence in a model-dependent approach. It is important to point out that decoherence is an effect which acts only on the quantum interference terms of the oscillation probabilities, while relaxation acts only on the constant terms, which allow flavor conversion even without mixing between the neutrino families.

For two-neutrino families [8], we have that the parameterization

$$
\tilde{V}_{k}=\sqrt{\gamma_{1}}\left(\begin{array}{cc}
\cos \delta_{\theta} & -\sin \delta_{\theta} \\
-\sin \delta_{\theta} & -\cos \delta_{\theta}
\end{array}\right),
$$

with $\delta_{\theta}=2(\tilde{\theta}-\theta)$, such that $\tilde{\theta}$ is the effective mixing angle in matter, leaves Eq. (B1) unchanged for any matter density.

As we can see, in a vacuum Eq. (B2) assumes the following form:

$$
V_{k}=\sqrt{\gamma_{1}}\left(\begin{array}{cc}
1 & 0 \\
0 & -1
\end{array}\right)
$$

since in a vacuum $\delta_{\theta}=0$.
To assure that the condition Eq. (B1) is satisfied for neutrinos propagating in both a vacuum and in constant density matter, it is shown in Ref. [8] that the operators $V_{k}$ must also transform when there is a change of basis. Therefore, we must have that

$$
\tilde{V}_{k}=U_{T}^{\dagger} V_{k} U_{T}=\sqrt{\gamma_{1}}\left(\begin{array}{cc}
1 & 0 \\
0 & -1
\end{array}\right)
$$

where $U_{T}=U^{\dagger} U_{M}$, and $U_{M}$ is the rotation matrix between the flavor basis and the effective mass basis, and, as we can see, it is equal to Eq. (B3).

When $V_{k}$ transform as Eq. (B4), the dissipator in Eq. (4) (where we consider only decoherence, not relaxation) is valid for neutrinos propagating in both a vacuum and in matter. Since the form is the same, the conditions for positivity are also the same for both cases, which assures that when Eqs. (5)-(7) are obeyed the physical meanings of the probabilities are guaranteed for oscillation both in a vacuum and in matter. It is also important to point out that, different from what is assumed by Ref. [16], in this work decoherence is assumed to be dependent on the matter density, as can be seen from Eq. (B2). More details of this discussion can be found in Ref. [8] for the case of two neutrinos.

It is worth noticing that, even though the calculations presented in Ref. [8] were made for the case of two neutrinos, the discussion of the concepts involved is very general, and its conclusions can be extended to the threeneutrino case.
[1] P. F. de Salas, D. V. Forero, C. A. Ternes, M. Tortola, and J. W. F. Valle, Phys. Lett. B 782, 633 (2018).

[2] R. Acciarri et al. (DUNE Collaboration), arXiv:1601.05471.

[3] R. Acciarri et al. (DUNE Collaboration), arXiv:1512.06148.

[4] J. Strait et al. (DUNE Collaboration), arXiv:1601.05823.

[5] R. Acciarri et al. (DUNE Collaboration), arXiv:1601.02984.

[6] LBNF/DUNE Collaboration, Long-Baseline Neutrino Facility (LBNF) and Deep Underground Neutrino Experiment (DUNE): Conceptual design report, Vol. 3, Annex 3 A: Beamline at the Near Site.

[7] R. L. N. Oliveira and M. M. Guzzo, Eur. Phys. J. C 73, 2434 (2013).

[8] M. M. Guzzo, P. C. de Holanda, and R. L. N. Oliveira, Nucl. Phys. B908, 408 (2016).

[9] G. Balieiro Gomes, M. M. Guzzo, P. C. de Holanda, and R. L. N. Oliveira, Phys. Rev. D 95, 113005 (2017).

[10] G. L. Fogli, E. Lisi, A. Marrone, D. Montanino, and A. Palazzo, Phys. Rev. D 76, 033006 (2007).

[11] R. L. N. Oliveira and M. M. Guzzo, Eur. Phys. J. C 69, 493 (2010).
[12] J. A. B. Coelho, W. A. Mann, and S. S. Bashar, Phys. Rev. Lett. 118, 221801 (2017).

[13] R. L. N. Oliveira, Eur. Phys. J. C 76, 417 (2016).

[14] J. A. B. Coelho and W. A. Mann, Phys. Rev. D 96, 093009 (2017).

[15] R. L. N. de Oliveira, M. M. Guzzo, and P. C. de Holanda, Phys. Rev. D 89, 053002 (2014).

[16] J. A. Carpio, E. Massoni, and A. M. Gago, Phys. Rev. D 97, 115017 (2018).

[17] P. Coloma, J. Lopez-Pavon, I. Martinez-Soler, and H. Nunokawa, Eur. Phys. J. C 78, 614 (2018).

[18] H. P. Breuer and F. Petruccione, The Theory of Open Quantum Systems (Oxford University Press, Oxford, 2002).

[19] J. R. Ellis, J. S. Hagelin, D. V. Nanopoulos, and M. Srednicki, Nucl. Phys. B241, 381 (1984).

[20] E. Akhmedov, J. Kopp, and M. Lindner, J. Cosmol. Astropart. Phys. 09 (2017) 017.

[21] Daya Bay Collaboration, Eur. Phys. J. C 77, 606 (2017).

[22] C. Cohen-Tannoudji, B. Diu, and F. Laloë, Quantum Mechanics (Wiley, New York, 1977). 
[23] P. Huber, M. Lindner, and W. Winter, Comput. Phys. Commun. 167, 195 (2005).

[24] P. Huber, J. Kopp, M. Lindner, M. Rolinec, and W. Winter, Comput. Phys. Commun. 177, 432 (2007).

[25] F. P. An et al. (Daya Bay Collaboration), Phys. Rev. D 95, 072006 (2017).

[26] K. Abe et al. (T2K Collaboration), Phys. Rev. D 96, 092006 (2017).
[27] A. M. Dziewonski and D. L. Anderson, Phys. Earth Planet. Interiors 25, 297 (1981).

[28] F. Stacey, Physics of the Earth (Wiley, New York, 1969).

[29] T. Alion et al. (DUNE Collaboration), arXiv:1606.09550.

[30] M. Masud, M. Bishai, and P. Mehta, Sci. Rep. 9, 352 (2019).

[31] G. Barenboim and N. E. Mavromatos, J. High Energy Phys. 01 (2005) 034. 